Cienc Tecn UTEQ (2017) 10(1) p 1-6 ISSN 1390-4051; e-ISSN 1390-4043 @(1)(9)

\title{
Comparación de un sistema de intensificación del cultivo de arroz (SICA) con sistemas tradicionales de siembra en la zona de Churute, Ecuador
}

\section{Comparing a system of rice intensification (SRI) with traditional cropping systems in Churute, Ecuador}

\author{
Esteban Alberto Ochoa Herrera ${ }^{1}$, Eduardo Ignacio Álava Hidalgo ${ }^{2,3},{ }^{\circ}$ Eduardo J. Chica Martínez ${ }^{1}$ \\ ${ }^{1}$ Universidad de Cuenca. Facultad de Ciencias Agropecuarias. Carrera de Ingeniería Agronómica, 12 de Octubre y Diego de \\ Tapia, Cuenca, Ecuador. agro_fresas@yahoo.com; •eduardo.chica@ucuenca.edu.ec \\ ${ }^{2}$ Escuela Superior Politécnica del Litoral, ESPOL, Facultad de Ciencias de la Vida, Campus Gustavo Galindo, Km 30.5 vía \\ Perimetral, P.O. Box09-01-5863, Guayaquil,Ecuador. eialava@espol.edu.ec \\ ${ }^{3}$ Universidad Agraria del Ecuador, UAE, Facultad de Medicina Veterinaria y Zootecnia, Av. 25 de Julio y Pio Jaramillo, P.O. \\ Box 09-04-100, Guayaquil, Ecuador.
}

Rec.: 25.10.2016. Acept.: 20.01.2017.

Publicado el 1 de junio de 2017

\section{Resumen}

$\mathrm{E}^{1}$ 1 sistema de intensificación del cultivo de arroz (SICA) es un conjunto de prácticas que según reportes permite incrementar significativamente el rendimiento del cultivo. Este estudio incluye un ensayo de campo para evaluar el efecto de la aplicación de un SICA y dos sistemas de siembra tradicionales (siembra por trasplante y al voleo) sobre los componentes del rendimiento (número de panículas, granos por panícula y peso de granos) del cultivo de arroz bajo condiciones ambientales del Litoral Sur de Ecuador. En el estudio, no se detectaron diferencias significativas en los componentes de rendimiento por área entre los tres sistemas evaluados, pero sí se registraron diferencias en el rendimiento por planta. Las plantas cultivadas usando SICA tuvieron un rendimiento por planta más alto, más macollos y más panículas por planta que las cultivadas usando los métodos tradicionales, lo que indica que el SICA favoreció el desarrollo morfológico y fisiológico de la planta durante el cultivo. Bajo las condiciones de este ensayo, el SICA no incrementó el rendimiento por área del cultivo, pero incrementó la eficiencia fisiológica de las plantas de forma individual.

Palabras clave: Oriza sativa, manejo tradicional, siembra al voleo, arroz de trasplante, riego.

\begin{abstract}
$\mathrm{T}$ he System of Rice Intensification (SRI) is a set of practices that reportedly increases yields in irrigated rice. Here, we report the results of a field trial conducted to evaluate an SRI and two paddy rice cultivation systems traditionally used in Ecuador (transplant and broadcast). No significant differences in yield components per area were detected among the three systems, however significant differences in yield components per plant were observed. SRI-cultivated plants produced higher yields per plant, more tillers and more panicles per plant than those cultivated using traditional systems, what indicates that SRI modified the morphological and physiological development of the plants under study. In this experiment, SRI did not increase the yield per area, but cultivation using SRI did increase the physiological efficiency of individual plants.
\end{abstract}

Key words: Oriza sativa, traditional crop management, broadcast planting, transplant rice, irrigation 


\section{Introducción}

$\mathrm{E}$ l sistema de intensificación del cultivo de arroz (SICA) es un conjunto flexible de prácticas diseñadas para incrementar la productividad del cultivo de arroz. Este sistema consiste en la siembra de arroz por trasplante, pudiéndose utilizar plántulas jóvenes $(<8$ días), una planta por sitio, un mayor espaciamiento entre plantas y la aplicación de riego de manera intermitente y sin inundación a fin de mantener condiciones aeróbicas constantes en el suelo. Inicialmente, el SICA fue propuesto por el sacerdote jesuita Henri de Laulanié para ayudar a productores de arroz empobrecidos del centro de Madagascar, ganando posteriormente apoyo a nivel mundial de productores, técnicos y organizaciones de desarrollo rural (De Laulanié, 2011). No obstante, el SICA ha recibido también serias críticas debido a la falta de repetitividad de los rendimientos extraordinarios reportados como resultado de su aplicación en varias partes de mundo (Sheehy et al., 2004; McDonald et al., 2006). Estas críticas han sido a su vez vehementemente rechazadas por los proponentes del SICA, y así, la discusión acerca de la efectividad del SICA como estrategia de mejora de la productividad del cultivo de arroz, aún es un debate controvertido (McDonald et al., 2008; Uphoff et al., 2008).

En Ecuador, el arroz se cultiva típicamente en sistemas poco tecnificados que producen rendimientos inferiores $\left(3.82 \mathrm{Mg} \mathrm{ha}^{-1}\right)$ en comparación a los regionales (5.09 $\mathrm{Mg} \mathrm{ha}^{-1}$ ) (IRRI, 2013). Tradicionalmente, el incremento en rendimiento de este cultivo ha estado asociado a la liberación de variedades mejoradas y al incremento de uso de fertilizantes. Esto, representa una limitación para la mayoría de productores debido al consecuente incremento de los costos de producción. En este contexto, prácticas como el SICA, representan alternativas atractivas que en teoría son capaces de mejorar el rendimiento del cultivo únicamente modificando prácticas culturales, sin incrementar el requerimiento de insumos.

El primer reporte de la aplicación del SICA en Ecuador proviene de un ensayo de campo en arroz utilizando tierras bajas de la zona de Daule, desarrollado por la Organización no Gubernamental Fundec en 2008 (SRI-Rice, 2015). Aquí, se reportaron rendimientos de granos de $8.8 \mathrm{Mg} \mathrm{ha}^{-1}$, sirviendo para iniciar su recomendación amplia a productores de otras zonas con condiciones semejantes (SRI-Rice, 2015). No obstante, al igual que en otras partes del mundo, estos resultados extremos deben ser interpretados con cautela debido a que usualmente provienen de experimentos que presentan deficiencias metodológicas que limitan su repetitividad (Sheehy et al., 2004). Esta falta de evaluación formal a su vez genera incertidumbre respecto a si la implementación de estas prácticas podría generar resultados positivos o negativos para los productores en una determinada zona (Moser \& Barrett, 2003). Así, el objetivo de este trabajo fue evaluar el efecto de la aplicación del SICA en los componentes productivos de un cultivo de arroz de inundación respecto a dos sistemas tradicionales de siembra. Este trabajo representa un primer paso dentro de un proceso de validación más amplio de la aplicabilidad del SICA para el sector arrocero de Ecuador.

\section{Materiales y métodos}

\section{E} l estudio se desarrolló en una hacienda comercial del sector Río Ruidoso de la zona de Churute $\left(02^{\circ} 24^{\prime} \mathrm{S}\right.$, $79^{\circ} 24^{\prime} \mathrm{W}$ ) del cantón Naranjal, provincia del Guayas, Ecuador. El sitio de ensayo está ubicado a una altitud de $14 \mathrm{msnm}$ y con un clima tropical de sabana con invierno seco de acuerdo a la clasificación de Köppen y Geiger (1928). La temperatura promedio anual es de $26.5^{\circ} \mathrm{C}$, con $91 \%$ de humedad relativa y una precipitación anual de $992.40 \mathrm{~mm}$. El ensayo se desarrolló entre noviembre de 2014 y febrero de 2015 correspondiente al inicio de la estación lluviosa. Las parcelas se ubicaron en un Inceptisol arcilloso, donde los últimos cinco años anteriores se cultivó arroz.

Se utilizó la variedad INIAP 15 por ser ampliamente cultivada por los productores de la zona. Esta variedad desarrollada por el Instituto Nacional Autónomo de Investigaciones Agropecuarias (INIAP), generalmente se cultiva bajo riego en la cuenca baja del río Guayas, con un ciclo de entre 117 y 128 días.

La preparación del terreno consistió en dos pases de arado de discos y fangueo, acorde a las técnicas utilizadas por los productores de la zona. Los tratamientos fueron los sistemas de cultivo al voleo, por trasplante tradicional y el Sistema de Intensificación del Cultivo de Arroz (SICA). Para el sistema por voleo, semillas pre-germinadas fueron sembradas a $90 \mathrm{kgha}^{-1}$ sobre el suelo saturado. Luego de la siembra, el suelo se mantuvo próximo a capacidad de campo hasta que la planta alcanzó una altura de $15 \mathrm{~cm}$, permaneciendo inundadas las parcelas con una lámina de $20 \mathrm{~mm}$ de agua hasta el final de la fase de llenado de grano (R6). Posteriormente, permanecieron sin riego hasta la cosecha.

Para el sistema de trasplante tradicional, se preparó un semillero sobre suelo utilizando semilla pregerminada. A los 25 días, las plantas fueron trasplantadas con seis plantas por sitio con un espaciamiento de $25 \times 25 \mathrm{~cm}$ entre sitios de acuerdo a la práctica de los 
productores de la zona. Estas parcelas permanecieron bajo inundación con una lámina de $20 \mathrm{~mm}$ de agua hasta el final de la fase de llenado de grano, permaneciendo sin riego hasta la cosecha.

Para el SICA, se preparó un semillero con semilla pre-germinada como se explicó para el sistema de trasplante tradicional, en este caso las plantas fueron trasplantadas a los ocho días desde su germinación y se sembró sólo una planta por sitio y con un espaciamiento entre sitios de $25 \times 25 \mathrm{~cm}$. El suelo en estas parcelas permaneció cerca de su capacidad de campo, pero no inundado durante la mayor parte del experimento excepto durante la fase de llenado de granos, las parcelas fueron inundadas con una lámina de $20 \mathrm{~mm}$ de agua. Posteriormente, fueron drenadas y permanecieron sin riego hasta la cosecha. El Cuadro 1 muestra el número de sitios, plantas por sitio y número de plantas por $\mathrm{m}^{2}$ registrados en cada uno de los tratamientos una vez establecido el ensayo. El manejo de las malezas, artrópodos y enfermedades se desarrolló de manera igual en todas las parcelas. Se realizaron dos controles químicos de malezas a los 18 y 25 días después del trasplante (ddt) para controlar malezas de hoja ancha y angosta, y poblaciones de Rotboellia cochinchinensis. Después de los 25 ddt el control de malezas se realizó exclusivamente de forma manual hasta el final del ciclo. En el cultivo se presentaron ataques de Tagosodes orizicolus, Rupella albinella, Diatraea sp. y Tibraca sp. que fueron controlados con la aplicación de insecticidas sintéticos. A los 80 ddt se registró manchado de grano por Helminthosporium sp. que fue controlado con una mezcla comercial de propiconazole y difeconazole.

\section{Variables evaluadas}

Los componentes del rendimiento (número de macollos, panículas, granos por panícula y peso del grano) fueron cuantificados al final del ensayo cosechando todas las plantas en 10 sitios de siembra dentro de un área de 1x1 m ubicada en el centro de cada parcela experimental. Estas plantas fueron cosechadas manualmente y se contó el número de plantas por sitio, macollos y panículas por planta y el número de granos llenos y vanos en cada panícula. Posteriormente, se

Cuadro 1. Número de sitios y población de plantas de arroz cultivadas usando SICA y sistemas de siembra tradicional por trasplante y voleo

\begin{tabular}{lccc}
\hline Tratamiento & Sitios $\cdot \mathbf{m}^{-2}$ & Plantas $\cdot \mathbf{s i t i o}^{-\mathbf{1}}$ & Plantas $\cdot \mathbf{m}^{-2}$ \\
\hline SICA & 16 & 1 & 16 \\
Trasplante & 16 & 6 & 96 \\
Voleo & $297 \pm 11$ & 1 & $297 \pm 11$ \\
\hline
\end{tabular}

recolectaron todos los granos de cada panícula, partes aéreas y raíces de estas plantas y fueron secados en un secador de aire forzado a $80^{\circ} \mathrm{C}$ hasta alcanzar el peso constante para determinar su peso seco. El rendimiento por $\mathrm{m}^{2}$ fue estimado multiplicando el rendimiento promedio de grano por planta por el número de plantas por $\mathrm{m}^{2}$ para cada sistema de producción.

\section{Análisis estadístico}

El experimento fue conducido usando un diseño de bloques completos al azar con 5 repeticiones. Cada unidad experimental estuvo conformada por una parcela de 10x10 m. Luego de probar la normalidad y homocedasticidad de los datos, estos fueron analizados a través de análisis de varianza usando el programa $R$. Para comparar las medias de los tratamientos se aplicó la prueba de Tukey $(\mathrm{p} \leq 0.05)$.

\section{Resultados y discusión}

$\mathrm{E}^{1}$ análisis estadístico no detectó diferencias significativas para la principal variable de comparación, el rendimiento por unidad de área (Figura 1). La falta de diferencias entre el SICA y los sistemas tradicionales contradicen el primer reporte comparativo entre el SICA y sistemas de producción tradicionales en Ecuador, en el cual se reportaron rendimientos 3.8 veces mayores en el SICA respecto al sistema de siembra tradicional (SRI-Rice, 2015). De la misma forma, estos resultados contradicen también reportes de Cuba en donde la aplicación del SICA incrementó significativamente el rendimiento de grano por hectárea (Maqueira, 2007). No obstante, los resultados obtenidos se alinean con reportes de Brasil, Panamá, India y China en donde el incremento de rendimiento del SICA respecto de sistemas tradicionales fue mucho más moderado $(\sim 10 \%)$ o incluso menor a los controles tradicionales (Turmel et al., 2011; Gehring et al., 2013; Kunnathadi et al., 2015; Wu, Ma y Uphoff, 2015).

A nivel internacional, varios meta-análisis de estudios comparativos entre el SICA y sistemas de producción tradicionales coinciden que, los reportes de rendimientos extraordinarios para el SICA son probablemente consecuencia de errores de medición o producto de interacciones específicas entre el sistema de cultivo y el ambiente donde se desarrolla el cultivo (Sheehy et al., 2004; McDonald et al., 2006; Turmel et al., 2011). En referencia a estas potenciales interacciones, existe una relación entre los mejores rendimientos obtenidos con la aplicación del SICA y el desarrollo del cultivo bajo condiciones marginales de producción, principalmente en suelos de baja fertilidad, 
esta relación sugiere que en zonas en las cuales el arroz es cultivado de manera intensiva en suelos de alta fertilidad (como en el caso de este experimento) el SICA generaría menores o nulos incrementos en el rendimiento (McDonald et al., 2006; Turmel et al., 2011).

Pese a no existir diferencias significativas para rendimiento por área, el rendimiento por planta sí mostró diferencias significativas $(\mathrm{p} \leq 0.05)$, siendo las plantas cultivadas bajo el SICA las de mayor promedio (Cuadro 2, Figura 2). De igual manera, bajo este sistema se presentó un mayor desarrollo vegetativo (peso seco y número de macollos por planta). No obstante, debido al menor número de macollos y panículas $\mathrm{m}^{-2}$ asociados a una menor densidad de siembra, en comparación con el sistema de siembra al voleo, los rendimientos por área en estos dos sistemas fueron similares. En el sistema SICA las plantas son más eficientes, presentando mayor rendimiento por planta, como resultado de una mayor acumulación de biomasa. La idea de una mayor eficiencia fisiológica de las plantas cultivadas en el SICA también está soportada por los rendimientos similares observados entre este sistema y el sistema de trasplante tradicional, en el cual los espaciamientos de siembra son iguales al SICA pero se trasplantaron seis veces más plantas por $\mathrm{m}^{2}$. Esta mayor eficiencia fisiológica implica también una mayor eficiencia en el uso de recursos que podría beneficiar al productor a través de menores costos de producción, debido al menor requerimiento de plantas (semilla), menor consumo de agua, y, consecuentemente, menores gastos por bombeo como ha sido reportado en Cuba (Martín et al., 2010). En el caso del requerimiento de semilla, en este experimento, el SICA requirió $1 / 6$ de las plantas utilizadas para el sistema de trasplante tradicional y $1 / 20$ de las plantas requeridas en el sistema de voleo; mientras que, en el requerimiento de riego, los gastos promedios de bombeo en el SICA fueron $40 \%$ de los registrados en el sistema de trasplante y $60 \%$ de los registrados en el sistema de voleo. En consecuencia, algunas prácticas derivadas del SICA podrían ser estudiadas con mayor detalle a fin de diseñar sistemas más eficientes de producción de arroz. Estos sistemas, a su vez, podría facilitar la introducción de otras tecnologías, como por ejemplo, sistemas de siembra directa mecanizada, el uso de híbridos élite y la agricultura de conservación (ur Rasool et al., 2013; Wang et al., 2014).

Analizando cada uno de los componentes de rendimientos evaluados, el incremento del rendimiento por planta en el SICA se deriva principalmente de un incremento en el número de macollos por planta (Figura 2). El número de macollos por planta reportado en este trabajo (18) fue notablemente menor a los valores reportados anteriormente para el SICA en Ecuador (44, SRI-Rice, 2015) pero similares a los reportados en otras partes del mundo (Gehring et al., 2013; Kunnathadi et al., 2015). En todos los sistemas, el número de panículas por planta $(15,80 ; 2,95$ y 2,00 para SICA, trasplante tradicional y voleo respectivamente; Figura 2) correspondió casi completamente al número de macollos por planta $(18,06 ; 3,06$ y 2,03 para SICA, trasplante y voleo, respectivamente), indicando que casi todos los macollos produjeron panículas. Por otra parte, las panículas producidas en plantas cultivadas bajo SICA tuvieron más granos llenos que las panículas de plantas cultivadas bajo el sistema de voleo, pero, el mismo número de granos que las panículas de plantas cultivadas bajo el sistema de trasplante tradicional (Cuadro 2).

El número de granos vanos por panícula tuvo la misma tendencia en los tres sistemas, así el porcentaje de vaneamiento fue similar en el SICA y sistema de trasplante tradicional (13\%) y notablemente superior en el sistema de voleo (19\%). Similares resultados se obtuvo en el peso de grano, en los tres sistemas. En general, el efecto del SICA sobre el número de panículas por planta, porcentaje de vaneamiento y peso de grano son similares a los registrados en Brasil e India de 16 y 23 panículas por planta y 8 a $13 \%$ de granos vanos, pero diferentes a los obtenidos en Cuba en donde se reportan cerca de 29 panículas por planta y $85 \%$ más granos llenos por panícula (Maqueira, 2007;
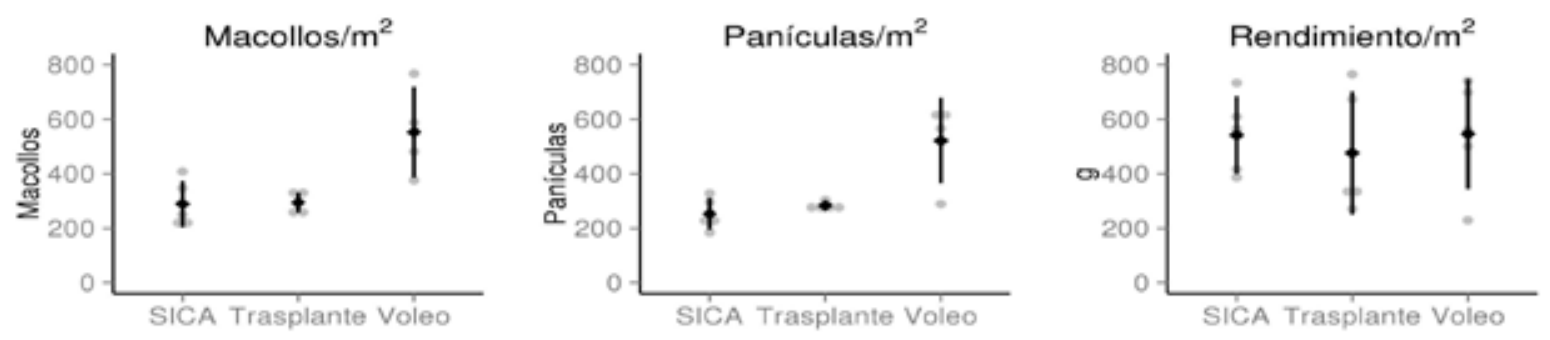

Figura 1. Número de macollos, panículas y rendimiento de grano por metro cuadrado en arroz cultivado usando SICA y sistemas de siembra tradicional por trasplante y voleo. El diamante y líneas negras representan a la media \pm la desviación estándar (n=5). Los círculos grises representan los datos originales. 
Cuadro 2. Altura de planta, peso seco, número de granos por panícula (llenos y vanos) y peso de grano en arroz cultivado usando SICA y sistemas de siembra tradicional por trasplante y voleo

\begin{tabular}{lccccc}
\hline \multicolumn{1}{c}{ Tratamiento } & $\begin{array}{c}\text { Altura de planta } \\
(\mathrm{cm})^{1}\end{array}$ & $\begin{array}{c}\text { Peso seco por } \\
\text { planta }(\mathrm{g})^{1}\end{array}$ & $\begin{array}{c}\text { Granos llenos } \\
\text { por panícula }^{1}\end{array}$ & $\begin{array}{c}\text { Granos vanos por } \\
\text { panícula }^{1}\end{array}$ & $\begin{array}{c}\text { Peso de grano } \\
(\mathrm{mg})^{1}\end{array}$ \\
\hline SICA & $91.92 \mathrm{a}$ & $1151.18 \mathrm{a}$ & $66.0 \mathrm{a}$ & $8.9 \mathrm{a}$ & $32.78 \mathrm{a}$ \\
Trasplante & $86.66 \mathrm{a}$ & $161.31 \mathrm{~b}$ & $48.2 \mathrm{ab}$ & $6.6 \mathrm{a}$ & $34.36 \mathrm{a}$ \\
Voleo & $79.13 \mathrm{a}$ & $74.06 \mathrm{~b}$ & $27.2 \mathrm{~b}$ & $5.2 \mathrm{a}$ & $33.44 \mathrm{a}$ \\
\hline
\end{tabular}
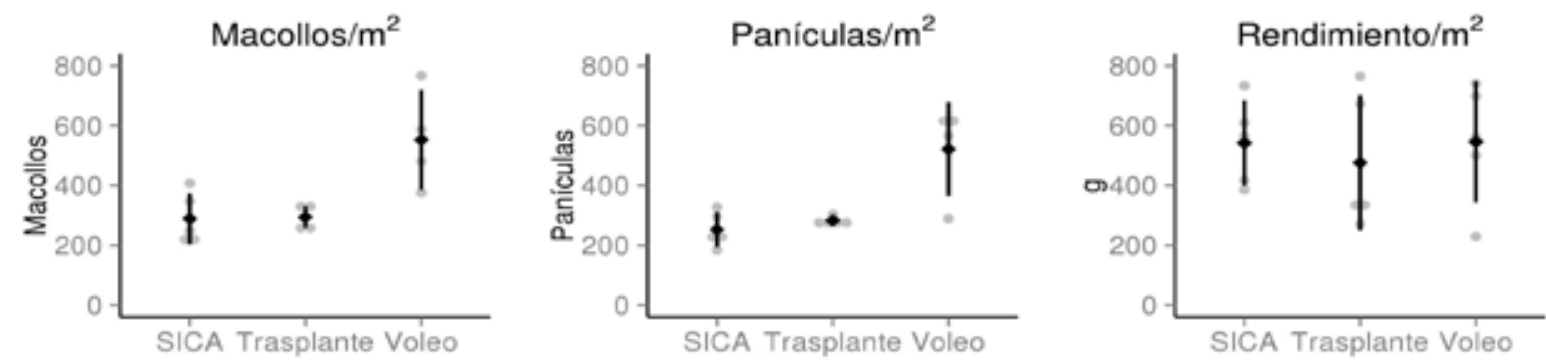

Figura 2. Número de macollos, panículas y rendimiento de grano por planta en arroz cultivado usando SICA y sistemas de siembra tradicional por trasplante y voleo. El diamante y líneas negras representan a la media \pm la desviación estándar (n=5). Los círculos grises representan los datos originales

Gehring et al., 2013; Kunnathadi et al., 2015).

En este estudio, no se detectaron diferencias en el rendimiento por unidad de área entre cultivos de arroz manejados bajo SICA y dos sistemas de siembra tradicionales en arroz de inundación. Una limitación de este estudio es que reporta resultados de un sólo experimento desarrollado en una sola temporada y en un solo ambiente. No obstante, este reporte es oportuno debido a los resultados obtenidos que contradicen reportes informales sobre rendimientos extraordinarios en cultivos de arroz manejados bajo SICA en Ecuador (SRI-Rice, 2015). El desarrollo del SICA a partir de procesos de desarrollo participativo ha marcado su diseminación a nivel mundial principalmente a través de asociaciones de productores, organismos no-gubernamentales e institutos de desarrollo rural. Infortunadamente, estas organizaciones basan sus recomendaciones en experimentos que no siempre han sido diseñados de manera que permitan una evaluación objetiva y sin sesgos de SICA (Sheehy et al., 2004; McDonald et al., 2008). Es interesante que, en contraste con los primeros reportes del SICA y la mayoría de reportes actuales en América Latina, en recientes meta-análisis realizados por autores promotores del SICA, los incrementos en rendimiento obtenidos por la implementación del SICA han sido fijados en niveles mucho más moderados $(\sim 25 \%)$ que son dependientes de condiciones locales relacionadas con el nivel de fertilidad de los suelos (Turmel et al., 2011; Wu et al., 2015). Esta especificidad entre condiciones locales de cultivo y el desempeño del SICA representa una de las principales limitaciones para recomendar de manera irrestricta la aplicación del SICA para todas las zonas arroceras. Consecuentemente, es necesario desarrollar ensayos de validación usando metodologías y diseños estandarizados en cada una de las zonas en donde se desee evaluar si el SICA representa o no una alternativa a los sistemas de producción tradicionales (Turmel et al., 2011).

\section{Conclusiones}

T a aplicación del SICA en un cultivo de arroz de Linundación produjo rendimientos por $\mathrm{m}^{2}$ similares a los dos sistemas tradicionales evaluados. No obstante, el rendimiento por planta fue significativamente mayor en el SICA, comparado a los otros dos sistemas. El mayor rendimiento de granos por planta observado con el SICA es una consecuencia de un mayor número de macollos. El mayor rendimiento de granos por planta, así como el menor requerimiento de plantas y de irrigación observado con el uso del SICA, indica que este sistema fue más eficiente en el empleo de recursos. 


\section{Bibliografía}

De Laulanié, H. (2011). Intensive rice farming in Madagascar. Tropicultura, 29(3), 183-187.

Gehring, C., de Moura, EG., Soares Santos, RR., Aguiar, A. das CF., de Sousa, AMB., \& Boddey, RM. (2013). Ecological intensification of rice production in the lowlands of Amazonia - Options for smallholder rice producers. European Journal of Agronomy, 46, 25-33.

IRRI. (2013). World Rice Statistics Online Query Facility. Recuperado 26 de diciembre de 2015, a partir de http://ricestat.irri.org:8080/wrs2/entrypoint.htm

Köppen, W.; Geiger, R. (1928). Klimate der Erde. Gotha: Verlag Justus Perthes. Wall-map $150 \mathrm{~cm} \times 200 \mathrm{~cm}$.

Kunnathadi, M., Abraham, C., Thomas, CG., \& Girija, T. (2015). Comparative evaluation of SRI with conventional system in the irrigated rice tracts of Kerala. Journal of Tropical Agriculture, 53(1), 8-16.

Maqueira, L., Torres, W., Díaz, G., \& Torres, K. (2007). Efecto del sistema intensivo del cultivo arrocero (SICA) sobre algunas variables del crecimiento y el rendimiento en una variedad de ciclo corto. Cultivos Tropicales, 28(2), 59-61.

Martín, Y., Rodríguez, YE., Morejón, R., \& Soto, F. (2010). El sistema intensivo de cultivo del arroz (SICA) disminuye la cantidad de semillas para la siembra, aumenta los rendimientos agrícolas y ahorra el agua de riego. Cultivos Tropicales, 31(1), 70-73.

McDonald, AJ., Hobbs, PR., \& Riha, SJ. (2006). Does the system of rice intensification outperform conventional best management?: A synopsis of the empirical record. Field Crops Research, 96(1), 3136.

McDonald, AJ., Hobbs, PR., \& Riha, SJ. (2008). Stubborn facts: Still no evidence that the System of Rice Intensification out-yields best management practices (BMPs) beyond Madagascar. Field Crops Research, 108(2), 188-191.
Moser, CM., \& Barrett, CB. (2003). The disappointing adoption dynamics of a yield-increasing, low external-input technology: the case of SRI in Madagascar. Agricultural Systems, 76(3), 10851100.

Sheehy, JE., Peng, S., Dobermann, A., Mitchell, PL., Ferrer, A., Yang, J., Huang, J. (2004). Fantastic yields in the system of rice intensification: fact or fallacy? Field Crops Research, 88(1), 1-8.

SRI-Rice. (2015). System of Rice Intensification Ecuador. Recuperado 26 de diciembre de 2015, a partir de http://sri.cals.cornell.edu/countries/ ecuador/index.html

Turmel, MS., Turner, BL., \& Whalen, JK. (2011). Soil fertility and the yield response to the System of Rice Intensification. Renewable Agriculture and Food Systems, 26(03), 185-192.

Uphoff, N., Kassam, A., \& Stoop, W. (2008). A critical assessment of a desk study comparing crop production systems: The example of the 'system of rice intensification' versus 'best management practice'. Field Crops Research, 108(1), 109-114.

ur Rasool, F., Habib, R., \& Bhat, M. (2013). Agronomic evaluation of rice (Oryza sativa L.) for plant spacings and seedlings per hill under temperate conditions. African Journal of Agricultural Research, 8(37), 4650-4653.

Wang, D., Chen, S., Wang, Z., Ji, C., Xu, C., Zhang, X., \& Chauhan, BS. (2014). Optimizing hill seeding density for high-yielding hybrid rice in a single rice cropping system in South China. PLoS ONE, 9(10), e109417.

Wu, W., Ma, B., \& Uphoff, N. (2015). A review of the system of rice intensification in China. Plant and Soil. 393: 361. doi:10.1007/s11104-015-2440-6 